\title{
Unsteady Incompressible Viscoelastic Flow of a Generalised Maxwell Fluid between Two Rotating Infinite Parallel Coaxial Circular Disks
}

\author{
Dhiman Bose, Uma Basu \\ Department Applied Mathematics, University of Calcutta, Kolkata, India \\ Email: dhimanbose09@gmail.com, ubappmath@caluniv.ac.in
}

Received March 23, 2013; revised April 24, 2013; accepted May 1, 2013

Copyright (C) 2013 Dhiman Bose, Uma Basu. This is an open access article distributed under the Creative Commons Attribution License, which permits unrestricted use, distribution, and reproduction in any medium, provided the original work is properly cited.

\begin{abstract}
The unsteady incompressible viscous flow of a Generalised Maxwell fluid between two coaxial rotating infinite parallel circular disks is studied by using the method of integral transforms. The motion of the fluid is created by the rotation of the upper and lower circular disks with different angular velocities. A fractional calculus approach is utilized to determine the velocity profile in series form in terms of Mittag-Leffler function. The influence of the fractional as well as the material parameters on the velocity field is illustrated graphically.
\end{abstract}

Keywords: Generalised Maxwell Fluid; Laplace Transform; Finite Fourier Sine Transform; Mittag-Leffler Function; Fractional Derivative

\section{Introduction}

The study of fluid flow between two parallel disks is of practical importance in many fields such as machine storage devices, computer devices, crystal growth processes, turbine engines, radial diffusers, lubrication, viscometry etc. The rotating disc problem was first formulated by von Kármán [1]. He considered the flow of a viscous incompressible fluid under the influence of a rotating disk. Later Cochran [2] obtained asymptotic solutions to the steady hydro-dynamic problem formulated by von Karman. It is found that the disc acts are like a centrifugal fan, the fluid near the disc being thrown radially outwards. Hossain and Rahman [3] studied the steady flow between two porous rotating discs in the presence of transverse magnetic field. Hossain and Wilson [4] investigated unsteady flow of viscous incompressible fluid with temperature-dependent viscosity due to a rotating disc in the presence of transverse magnetic field and heat transfer. Wenchang, Wenxiao and Mingyu [5] have studied unsteady flows of a visco-elastic fluid with the fractional Maxwell model between two parallel plates. Maji, Ghara, Jana and Das [6] have considered unsteady MHD flow between two eccentric rotating disks. Liu, Zheng, Zhang and Zong [7] discussed the oscillating flows and heat transfer of a Generalised Oldroyed-B fluid in the presence of magnetic field. Kempegowda and Balagon- dar [8] have worked out exact solutions of non- Newtonian fluid flow between two moving parallel disks with stability analysis.

In the present paper we have considered unsteady incompressible visco-elastic flow of a generalized Maxwell fluid between two rotating infinite coaxial circular disks. In the aforesaid problems, time derivative of integer order has been considered in the Navier-Stokes equation but in the present problem we have considered the constitutive equation for Maxwell fluid with fractional order time derivative instead of integer order time derivative. In the constitutive equation the time derivative of integer order is replaced by the Caputo fractional calculus operator. We have obtained the analytical solution to the velocity field in series involving Mittag-Leffler function and illustrated graphically the dependence of the velocity field on the fractional and material parameters.

\section{Generalised Maxwell Model and Basic Equation}

The constitutive equation of a Generalised incompressible Maxwell fluid can be written as,

$$
\tau+\lambda^{\alpha} D_{t}^{\alpha} \tau=G \lambda^{\beta} D_{t}^{\beta} \varepsilon
$$

where, $\tau$ is the shear stress, $\lambda=\frac{\mu}{G}$ is a relaxation 
parameter, $G$ is the shear modulus, $\alpha$ and $\beta$ are fractional parameters such that $0 \leq \alpha \leq \beta \leq 1$ and $\varepsilon$ is the shear strain. $D_{t}^{\alpha}$ and $D_{t}^{\beta}$ are Caputo operators given by

$$
D_{t}^{\alpha} f(t)=\frac{1}{\Gamma(1-p)} \int_{0}^{t} \frac{f^{\prime}(u)}{(t-u)^{p}} \mathrm{~d} u, 0 \leq p<1
$$

For $\alpha=\beta=1$ the Equation (1) gives Ordinary Maxwell fluid model and for $\alpha=0, \beta=1$, a Classical Newtonian fluid model is recovered.

The Equation (1) can be rewritten as

$$
\tau+\lambda^{\alpha} D_{t}^{\alpha} \tau=G \lambda^{\beta} D_{t}^{\beta-1} \frac{\mathrm{d} \varepsilon}{\mathrm{d} t}
$$

where $\frac{\mathrm{d} \varepsilon}{\mathrm{d} t}$ is the shear rate.

The equation of motion in the absence of the body force can be written as

$$
\rho \frac{D \mathbf{V}}{D t}=\nabla \cdot \tau
$$

where $\rho$ is the density of the fluid, $\mathbf{V}$ is the fluid velocity, $\frac{D}{D t}$ is the material derivative, $\tau$ is the stress tensor.

The equation of continuity is given by

$$
\nabla \cdot \mathbf{V}=0
$$

\section{Mathematical Formulation}

Let an incompressible viscous Generalised Maxwell fluid be bounded by two coaxial infinite parallel circular disks at a distance " $d$ " apart and the fluid as well as the disks are initially at rest as shown in Figure 1. Suddenly the lower and the upper disks begin to rotate with velocities $\Omega$ and $\mathrm{s} \Omega$ respectively and as a consequence the fluid is set in motion. Here we take the cylindrical coordinate system $(r, \theta, z)$, where $r, \theta$ and $z$-coordinates are taken in the radial, cross radial and the direction joining the centers of the circular disks. We take the velocity profile of the form $\mathbf{V}=v(z, t) e_{\theta}$ where $e_{\theta}$ is the unit vector in the $\theta$-direction. For such flows the constraint of incompressibility is automatically satisfied. For this problem the constitutive relationship becomes

$$
\tau_{z \theta}+\lambda^{\alpha} \frac{\partial^{\alpha}}{\partial t^{\alpha}} \tau_{z \theta}=G \lambda^{\beta} \frac{\partial^{\beta-1}}{\partial t^{\beta-1}}\left(\frac{\partial v}{\partial z}\right)
$$

The momentum equation is

$$
\rho \frac{\partial v}{\partial t}=\frac{\partial \tau_{z \theta}}{\partial z}
$$

Eliminating $\tau_{z \theta}$ between the Equations (6) and (7) we get the basic equation as

$$
\rho \frac{\partial v}{\partial t}+\rho \lambda^{\alpha} \frac{\partial^{\alpha}}{\partial t^{\alpha}}\left(\frac{\partial v}{\partial t}\right)=G \lambda^{\beta} \frac{\partial^{\beta-1}}{\partial t^{\beta-1}}\left(\frac{\partial^{2} v}{\partial z^{2}}\right)
$$

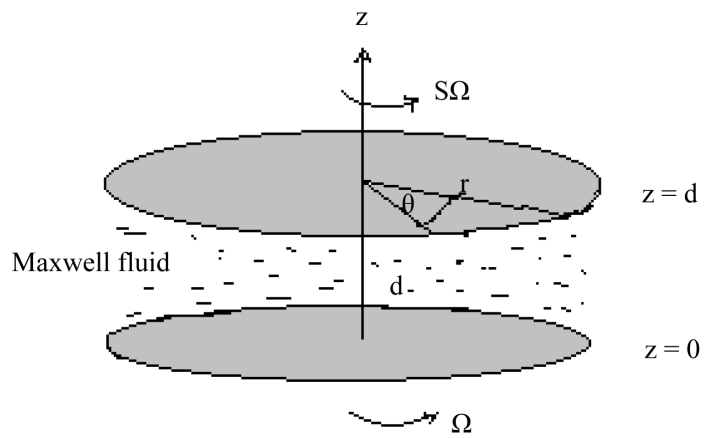

Figure 1. Geometry of the problem.

The Equation (8) is the governing equation of the flow of a Generalised Maxwell fluid between two rotating infinite parallel circular disks considered in the present problem.

The boundary conditions are given by

$$
\begin{gathered}
u=0, v=r \Omega, w=0 \text { at } z=0 \\
u=0, v=s r \Omega, w=0 \text { at } z=d
\end{gathered}
$$

" $s$ " is some constant.

The initial condition is given by

$$
v(z, 0)=0,0<z<d
$$

Now let us introduce the dimensionless variables

$$
v^{\prime}=\frac{v}{r \Omega}, z^{\prime}=\frac{z}{d}, t^{\prime}=\frac{t \Omega^{2} d^{2} \rho}{\mu}
$$

Then the governing Equation (8) in non-dimensional variables is given by (for simplicity the dimensionless mark "'" will be neglected hereinafter).

$$
\frac{\partial v}{\partial t}+\eta^{\alpha} \frac{\partial^{\alpha+1} v}{\partial t^{\alpha+1}}=\varsigma \eta^{\beta-1} \frac{\partial^{\beta-1}}{\partial t^{\beta-1}}\left(\frac{\partial^{2} v}{\partial z^{2}}\right)
$$

where, $\eta=\frac{\rho d^{2} \Omega^{2}}{G}, \varsigma=\frac{\mu^{2}}{\rho^{2} d^{4} \Omega^{2}}$

The boundary conditions in non-dimensional variable becomes

$$
v(0, t)=1 \text { and } v(1, t)=s
$$

Let us consider the transformation given by

$$
v^{*}(z, t)=v(z, t)-(1-z)
$$

Then in terms of new variable the governing equation becomes

$$
\frac{\partial v^{*}}{\partial t}+\eta^{\alpha} \frac{\partial^{\alpha+1} v^{*}}{\partial t^{\alpha+1}}=\varsigma \eta^{\beta-1} \frac{\partial^{\beta-1}}{\partial t^{\beta-1}}\left(\frac{\partial^{2} v^{*}}{\partial z^{2}}\right)
$$

Subject to the boundary conditions

$$
v^{*}(0, t)=0 \text { and } v^{*}(1, t)=s
$$

and initial condition 


$$
v^{*}(z, 0)=z-1
$$

Taking Laplace transformation and using initial condition we get from Equation (11)

$$
\begin{aligned}
& \varsigma \eta^{\beta-1} p^{\beta-1} \frac{\mathrm{d} V^{*}(z, p)}{\mathrm{d} z^{2}} \\
& =\left(\eta^{\alpha} p^{\alpha+1}+p\right) V^{*}(z, p)-(z-1)\left(1+p^{\alpha} \eta^{\alpha}\right)
\end{aligned}
$$

where, $V^{*}(z, p)$ is the Laplace transformation of $V^{*}(z, p)$ defined by

$$
V^{*}(z, p)=\int_{0}^{\infty} \exp (-p t) v^{*}(z, t) \mathrm{d} t
$$

where " $p$ " is Laplace transform parameter.

Taking finite Fourier sine transformation we get from the Equation (12)

$$
\begin{aligned}
& \varsigma \eta^{\beta-1} p^{\beta-1} \int_{0}^{1} \frac{\mathrm{d}^{2} V^{*}(z, p)}{\mathrm{d} z^{2}} \sin (n \pi z) \mathrm{d} z \\
& =\left(\eta^{\alpha} p^{\alpha+1}+p\right) \bar{V}_{s}^{*}(n, p) \\
& +\left(1+p^{\alpha} \eta^{\alpha}\right) \int_{0}^{1}(1-z) \sin (n \pi z) \mathrm{d} z
\end{aligned}
$$

where, $\bar{V}_{s}^{*}(n, p)$ is the finite Fourier sine transformation of $V^{*}(z, p)$ defined by

$$
\bar{V}_{s}^{*}(n, p)=\int_{0}^{1} V^{*}(z, p) \sin (n \pi z) \mathrm{d} z
$$

where $n=1,2,3, \cdots$

Taking Laplace transformation of the boundary conditions we get,

$$
V^{*}(0, p)=0 \text { and } V^{*}(1, p)=\frac{s}{p}
$$

Using the above conditions we get from Equation (13)

$$
\begin{aligned}
\bar{V}_{s}^{*}(n, p) & =\frac{(-1)^{n+1} s \varsigma \eta^{\beta-1} p^{\beta-2} n \pi}{\eta^{\alpha} p^{\alpha+1}+p+\varsigma \eta^{\beta-1} p^{\beta-1}(n \pi)^{2}} \\
& -\frac{1+\eta^{\alpha} p^{\alpha}}{n \pi} \times \frac{1}{\eta^{\alpha} p^{\alpha+1}+p+\varsigma \eta^{\beta-1} p^{\beta-1}(n \pi)^{2}}
\end{aligned}
$$

The Equation (14) can be written as

$$
\begin{aligned}
& \bar{V}_{s}^{*}(n, p) \\
& =(-1)^{n+1} s \varsigma \eta^{\beta-1-\alpha} n \pi \frac{\frac{p^{-1}}{p^{\alpha-\beta+2}+\varsigma \eta^{\beta-\alpha-1}(n \pi)^{2}}}{1+\frac{p^{2-\beta} \eta^{-\alpha}}{p^{\alpha-\beta+2}+\varsigma \eta^{\beta-\alpha-1}(n \pi)^{2}}} \\
& -\frac{1+\eta^{\alpha} p^{\alpha}}{n \pi} \frac{\frac{\eta^{-\alpha} p^{1-\beta}}{1+\frac{p^{\alpha-\beta+2}+\varsigma \eta^{\beta-\alpha-1}(n \pi)^{2}}{p^{2-\beta} \eta^{-\alpha}}}}{p^{\alpha-\beta+2}+\varsigma \eta^{\beta-\alpha-1}(n \pi)^{2}}
\end{aligned}
$$

In order to avoid the lengthy procedure of residues and contour integrals, we rewrite the Equation (15) into series form given by

$$
\begin{aligned}
& \bar{V}_{s}^{*}(n, p) \\
& =(-1)^{n+1} s \varsigma \eta^{\beta-1-\alpha} n \pi \sum_{k=0}^{\infty}(-1)^{k} \frac{p^{k(2-\beta)-1} \eta^{-k \alpha}}{\left(p^{\alpha-\beta+2}+\varsigma \eta^{\beta-\alpha-1}(n \pi)^{2}\right)^{k+1}} \\
& -\frac{p^{\alpha}+\eta^{-\alpha}}{n \pi} \sum_{k=0}^{\infty}(-1)^{k} \frac{p^{k(2-\beta)+(1-\beta)} \eta^{-(k+1) \alpha}}{\left(p^{\alpha-\beta+2}+\varsigma \eta^{\beta-\alpha-1}(n \pi)^{2}\right)^{k+1}}
\end{aligned}
$$

Now we have an important Laplace transformation of the nth order derivative of Mittag-Leffler function $E_{\alpha, \lambda}(y)$ given by

$$
\begin{aligned}
& \int_{0}^{\infty} \exp (-p t) t^{\alpha n+\lambda-1} E_{\alpha, \lambda}^{(n)}\left(-a t^{\alpha}\right) \mathrm{d} t \\
& =\frac{n ! p^{\alpha-\lambda}}{\left(p^{\alpha}+a\right)^{n+1}} \\
& E_{\alpha, \lambda}^{(n)}(y)=\frac{\mathrm{d}^{n}}{\mathrm{~d} y^{n}} E_{\alpha, \lambda}(y) \\
& =\sum_{j=0}^{\infty} \frac{(j+n) ! y^{j}}{j ! \Gamma(\alpha j+\alpha n+\lambda)}
\end{aligned}
$$

Taking inverse Laplace transformation we get from Equation (16)

$$
\begin{aligned}
V_{s}^{*}(n, t)= & (-1)^{n+1} s \varsigma \eta^{\beta-1-\alpha} n \pi \sum_{k=0}^{\infty}(-1)^{k} \eta^{-k \alpha} \frac{t^{(\alpha-\beta+2) k+\alpha-\beta+2-k(2-\beta)}}{k !} E_{\alpha-\beta+2, \alpha-\beta+3-k(2-\beta)}^{(k)}\left(-\varsigma \eta^{\beta-\alpha-1}(n \pi)^{2} t^{\alpha-\beta+2}\right) \\
& -\frac{1}{n \pi} \sum_{k=0}^{\infty}(-1)^{k} \eta^{-(k+1) \alpha} \frac{t^{(\alpha-\beta+2) k-k(2-\beta)}}{k !} E_{\alpha-\beta+2, \alpha+1-k(2-\beta)}^{(k)}\left(-\varsigma \eta^{\beta-\alpha-1}(n \pi)^{2} t^{\alpha-\beta+2}\right) \\
& -\frac{1}{n \pi} \sum_{k=0}^{\infty}(-1)^{k} \eta^{-(k+2) \alpha} \frac{t^{(\alpha-\beta+2) k+\alpha-k(2-\beta)}}{k !} E_{\alpha-\beta+2, \alpha+1-k(2-\beta)}^{(k)}\left(-\varsigma \eta^{\beta-\alpha-1}(n \pi)^{2} t^{\alpha-\beta+2}\right)
\end{aligned}
$$

Taking inverse finite Fourier sine transformation we get from Equation (19) 


$$
\begin{aligned}
v^{*}(z, t)= & 2 \sum_{n=1}^{\infty}(-1)^{n+1} s \varsigma \eta^{\beta-1-\alpha} n \pi \sin (n \pi z) \\
& \times \sum_{k=0}^{\infty}(-1)^{k} \eta^{-k \alpha} \frac{t^{(\alpha-\beta+2) k+\alpha-\beta+2-k(2-\beta)}}{k !} E_{\alpha-\beta+2, \alpha-\beta+3-k(2-\beta)}^{(k)}\left(-\varsigma \eta^{\beta-\alpha-1}(n \pi)^{2} t^{\alpha-\beta+2}\right) \\
& -2 \sum_{n=1}^{\infty} \frac{\sin (n \pi z)}{n \pi} \sum_{k=0}^{\infty}(-1)^{k} \eta^{-(k+1) \alpha} \frac{t^{(\alpha-\beta+2) k-k(2-\beta)}}{k !} E_{\alpha-\beta+2,1-k(2-\beta)}^{(k)}\left(-\varsigma \eta^{\beta-\alpha-1}(n \pi)^{2} t^{\alpha-\beta+2}\right) \\
& -2 \sum_{n=1}^{\infty} \frac{\sin (n \pi z)}{n \pi} \sum_{k=0}^{\infty}(-1)^{k} \eta^{-(k+2) \alpha} \frac{t^{(\alpha-\beta+2) k+\alpha-k(2-\beta)}}{k !} E_{\alpha-\beta+2, \alpha+1-k(2-\beta)}^{(k)}\left(-\varsigma \eta^{\beta-\alpha-1}(n \pi)^{2} t^{\alpha-\beta+2}\right)
\end{aligned}
$$

Changing the variable $v^{*}(z, t)$ to $v(z, t)$ by the transformation $v^{*}(z, t)=v(z, t)-(1-z)$ we get the expression for the velocity field as follows,

$$
\begin{aligned}
v(z, t)= & 1-z+2 \sum_{n=1}^{\infty}(-1)^{n+1} s \varsigma \eta^{\beta-1-\alpha} n \pi \sin (n \pi z) \\
& \times \sum_{k=0}^{\infty}(-1)^{k} \eta^{-k \alpha} \frac{t^{(\alpha-\beta+2) k+\alpha-\beta+2-k(2-\beta)}}{k !} E_{\alpha-\beta+2, \alpha-\beta+3-k(2-\beta)}^{(k)}\left(-\varsigma \eta^{\beta-\alpha-1}(n \pi)^{2} t^{\alpha-\beta+2}\right) \\
& -2 \sum_{n=1}^{\infty} \frac{\sin (n \pi z)}{n \pi} \sum_{k=0}^{\infty}(-1)^{k} \eta^{-(k+1) \alpha} \frac{t^{(\alpha-\beta+2) k-k(2-\beta)}}{k !} E_{\alpha-\beta+2,1-k(2-\beta)}^{(k)}\left(-\varsigma \eta^{\beta-\alpha-1}(n \pi)^{2} t^{\alpha-\beta+2}\right) \\
& -2 \sum_{n=1}^{\infty} \frac{\sin (n \pi z)}{n \pi} \sum_{k=0}^{\infty}(-1)^{k} \eta^{-(k+2) \alpha} \frac{t^{(\alpha-\beta+2) k+\alpha-k(2-\beta)}}{k !} E_{\alpha-\beta+2, \alpha+1-k(2-\beta)}^{(k)}\left(-\varsigma \eta^{\beta-\alpha-1}(n \pi)^{2} t^{\alpha-\beta+2}\right)
\end{aligned}
$$

\section{Limiting Cases}

Case-I If $\alpha=1, \beta=1$ then the equation of motion is

$$
\frac{\partial v(z, t)}{\partial t}+\eta \frac{\partial^{2} v(z, t)}{\partial t^{2}}=\varsigma \frac{\partial^{2} v(z, t)}{\partial z^{2}}
$$

Subject to the boundary conditions

$$
v(0, t)=1 \text { and } v(1, t)=s
$$

and initial condition

$$
v(z, 0)=0
$$

The Equation (21) is the damped wave equation and it represents the governing equation of an Ordinary Maxwell fluid.

Then we get the velocity profile from the Equation (20) as

$$
\begin{aligned}
& v_{\text {OMF }}(z, t) \\
& =1-z+2 \sum_{n=1}^{\infty}(-1)^{n+1} s \varsigma \eta^{-1} n \pi \sin (n \pi z) \\
& \quad \times \sum_{k=0}^{\infty}(-1)^{k} \eta^{-k} \frac{t^{k+2}}{k !} E_{2,3-k}^{(k)}\left(-\varsigma \eta^{-1}(n \pi)^{2} t^{2}\right) \\
& -2 \sum_{n=1}^{\infty} \frac{\sin (n \pi z)}{n \pi} \sum_{k=0}^{\infty}(-1)^{k} \eta^{-(k+1)} \frac{t^{k}}{k !} E_{2,1-k}^{(k)}\left(-\varsigma \eta^{-1}(n \pi)^{2} t^{2}\right) \\
& -2 \sum_{n=1}^{\infty} \frac{\sin (n \pi z)}{n \pi} \sum_{k=0}^{\infty}(-1)^{k} \eta^{-(k+2)} \frac{t^{k+1}}{k !} E_{2,2-k}^{(k)}\left(-\varsigma \eta^{-1}(n \pi)^{2} t^{2}\right)
\end{aligned}
$$

Case-II If $\alpha=0, \beta=1$, the equation of motion is given by

$$
\frac{\partial v(z, t)}{\partial t}=\frac{\varsigma}{2} \frac{\partial^{2} v(z, t)}{\partial z^{2}}
$$

subject to the boundary condition

$$
v(0, t)=1 \text { and } v(1, t)=s
$$

and initial condition

$$
v(z, 0)=0
$$

The Equation (23) is diffusion equation and it represents the governing equation of a Classical Newtonian Fluid.

Then we get the velocity profile from the Equation (20) as

$$
\begin{aligned}
& v_{C N F}(z, t) \\
& =1-z+2 \sum_{n=1}^{\infty}(-1)^{n+1} s \varsigma(n \pi) \sin (n \pi z) \\
& \quad \times \sum_{k=0}^{\infty}(-1)^{k} \frac{t}{k !} E_{1,2-k}^{(k)}\left(-\varsigma(n \pi)^{2} t\right) \\
& \quad-4 \sum_{n=1}^{\infty} \frac{\sin (n \pi z)}{n \pi} \sum_{k=0}^{\infty}(-1)^{k} \frac{1}{k !} E_{1,1-k}^{(k)}\left(-\varsigma(n \pi)^{2} t\right)
\end{aligned}
$$

\section{Conclusions and Numerical Results}

In the present paper we have found out the analytical solution to the velocity field by integral transform in series form in terms of Mittage-Leffler function for the unsteady incompressible flow of a Generalised Maxwell fluid between two rotating infinite parallel coaxial circular disks. We have got the solutions to the velocity fields 
for ordinary Maxwell fluid and Classical Newtonian fluid as the limiting cases of the solution of Generalised Maxwell fluid. In the constitutive equation for the Maxwell fluid the time derivative of integer order is replaced by Riemann-Liouville operator. The dependence of the velocity field on the fractional as well as material parameters has been illustrated graphically.

In Figure 2 the velocity is depicted against the distance from the lower disk along the direction of the common axis of rotation for different values of the fractional calculus parameter $\alpha$. From the Figure it is evident that as the value of the parameter $\alpha$ increases the fluid velocity in the $\theta$-direction increases. It can be noticed that the point of maximum velocity of the velocity curve gradually shifts towards the lower disk as $\alpha$ increases. In Figure 3 the velocity is plotted against the distance from the lower disk for different values of fractional calculus parameter $\beta$. As the value of $\beta$ increases, the fluid velocity increases near the lower disk whereas the velocity decreases near the upper disk. The velocity is depicted against the distance from the lower disk for different values of parameters " $\alpha$ " and " $s$ " in Figure 4. As " $\alpha$ " and " $s$ " increase simultaneously the fluid velocity increases and is maximum near the midpoint region between the two parallel disks. It is evident from the figure that the points of maximum velocity of the velocity curve gradually shift towards the lower disk as in the case in Figure 1.

In Figure 5 the velocity is plotted against the distance from the lower disk for different values of the material parameter " $\zeta$ ". It can be observed that as " $\zeta$ " increases the fluid velocity decreases near the lower disk whereas the velocity increases near the upper disk. The velocity is depicted against the distance from the lower disk for dif-

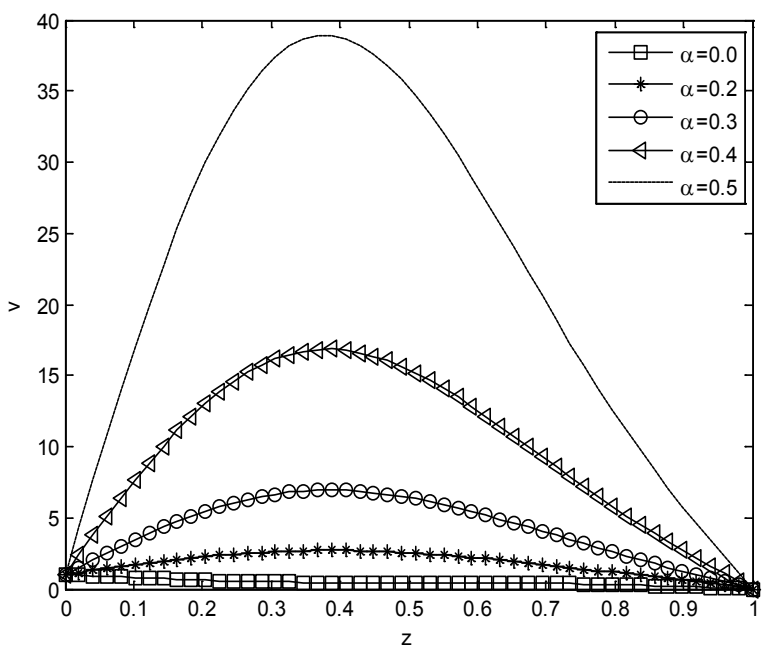

Figure 2. The velocity profile is depicted against the distance from the lower disk along the direction of the common axis of rotation for different values of fractional parameter $\alpha . \beta=1.0, \zeta=0.03, \eta=0.02, s=0.2, t=0.2$.

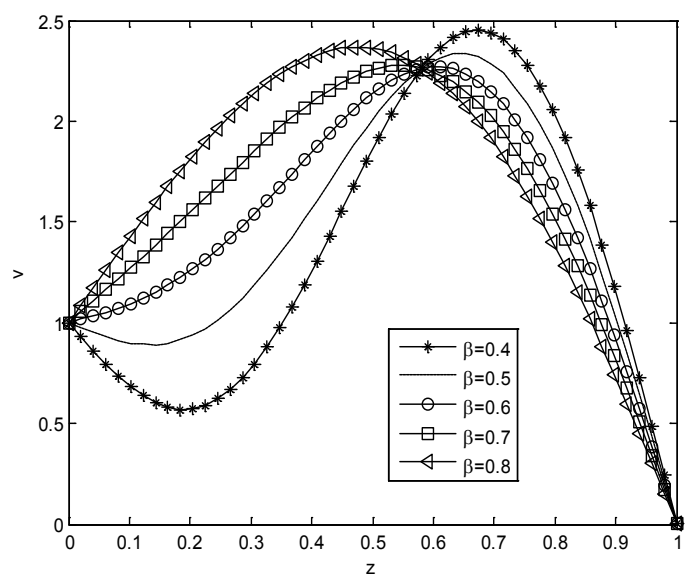

Figure 3. The velocity profile is depicted against the distance from the lower disk along the direction of the common axis of rotation for different values of fractional parameter $\beta . \alpha=0.2, \zeta=0.03, \eta=0.02, s=0.2, t=0.2$.

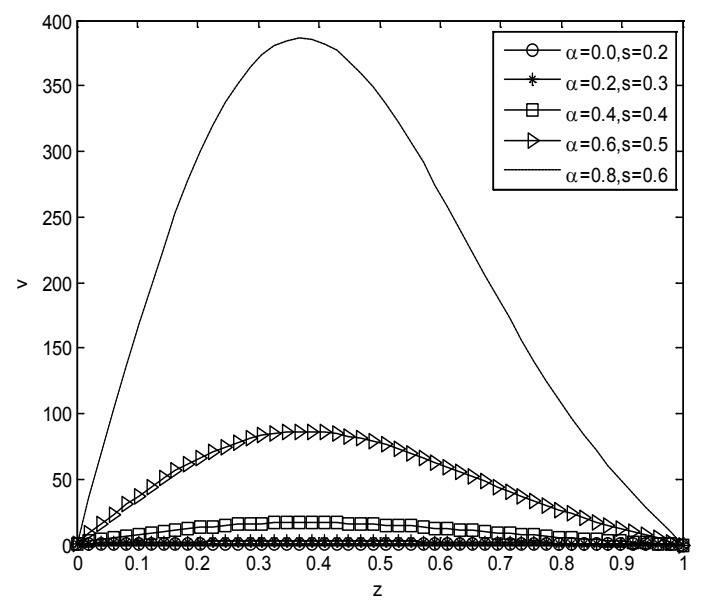

Figure 4. The velocity profile is depicted against the distance from the lower disk along the direction of the common axis rotation for different values of parameters $\alpha$ and $s$. $\beta=1.0, \zeta=0.03, \eta=0.02, t=0.2$.

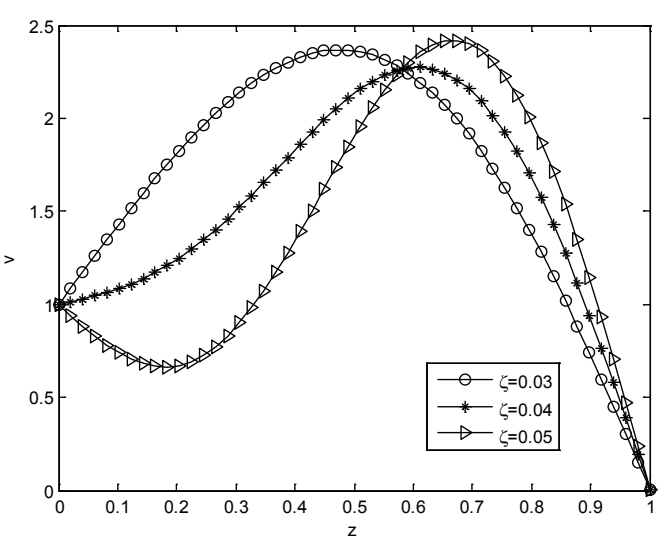

Figure 5. The velocity profile is depicted against the distance from the lower disk along the common axis of rotation for different values of the parameter $\zeta . \alpha=0.2, \beta=0.8$, $\eta=0.02, s=0.2, t=0.2$. 
ferent values of material parameter " $\eta$ " in Figure 6. It can be noticed that the velocity decreases with the increasing values of the parameter " $~ \eta$ " and the point of maximum velocity of the curve shifts towards the lower disks. The velocities are maximum near the lower disk for the cases. In Figure 7, as " $z$ " increases from 0 to 0.5 the fluid velocity decreases negatively whereas the velocity increases positively as " $z$ " increases from 0.6 to 1.0 . The velocity is depicted against the distance from the lower disk for three different cases namely Case-I Ordinary Maxwell Fluid, Case-II Classical Newtonian Fluid and Case III Generalised Maxwell fluid in Figure 8. In CaseI $\alpha \rightarrow 1, \beta \rightarrow 1$ and in Case-II $\alpha \rightarrow 0, \beta \rightarrow 1$ and in Case-III $\alpha=0.2, \beta=0.8$. In the case for Ordinary Maxwell Fluid, there is a point of local maximum (near

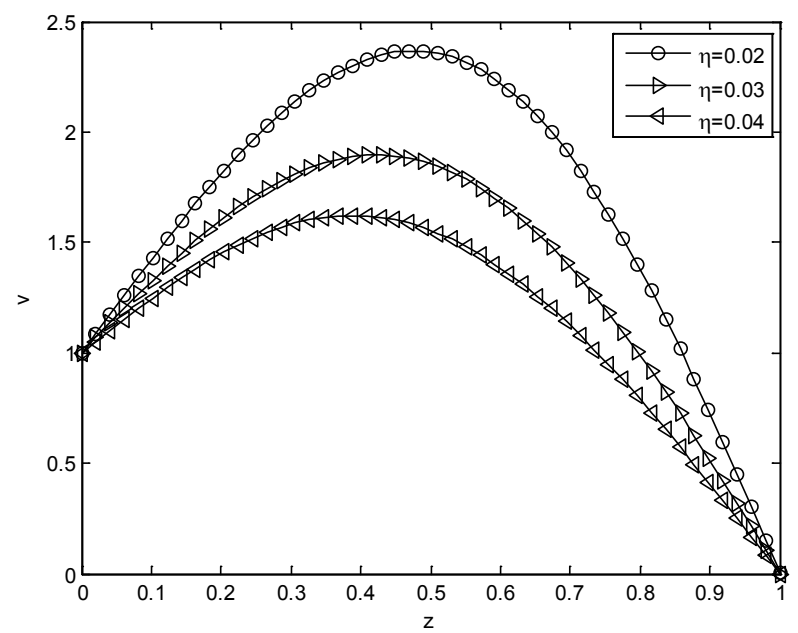

Figure 6. The velocity profile is depicted against the distance from the lower disk along the direction of the common axis of rotation for different values of $\eta . \alpha=0.2, \beta=0.8$, $\zeta=0.03, s=0.2, t=0.2$.

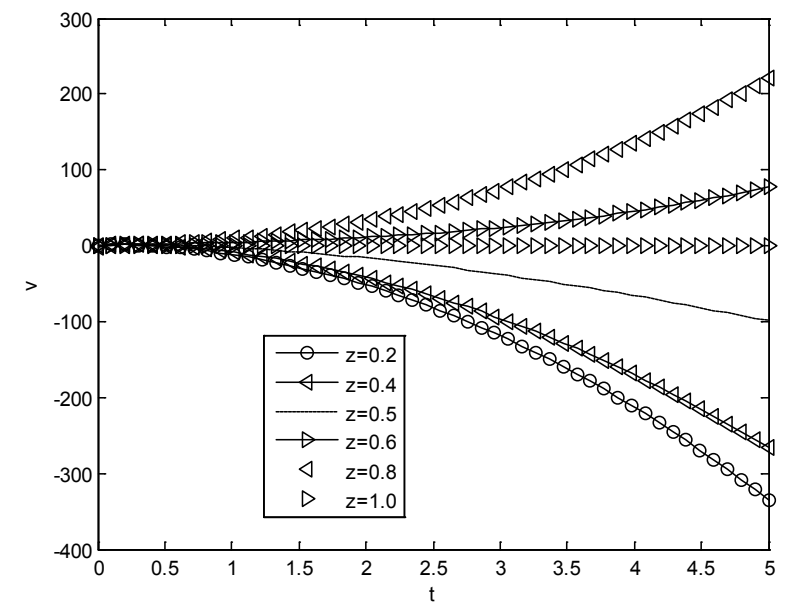

Figure 7. The velocity profile is depicted against time with different distances from the lower disk along the direction of the common axis of rotation. $\alpha=0.2, \beta=0.8, \zeta=0.03, \eta=$ $0.04, s=0.2$.

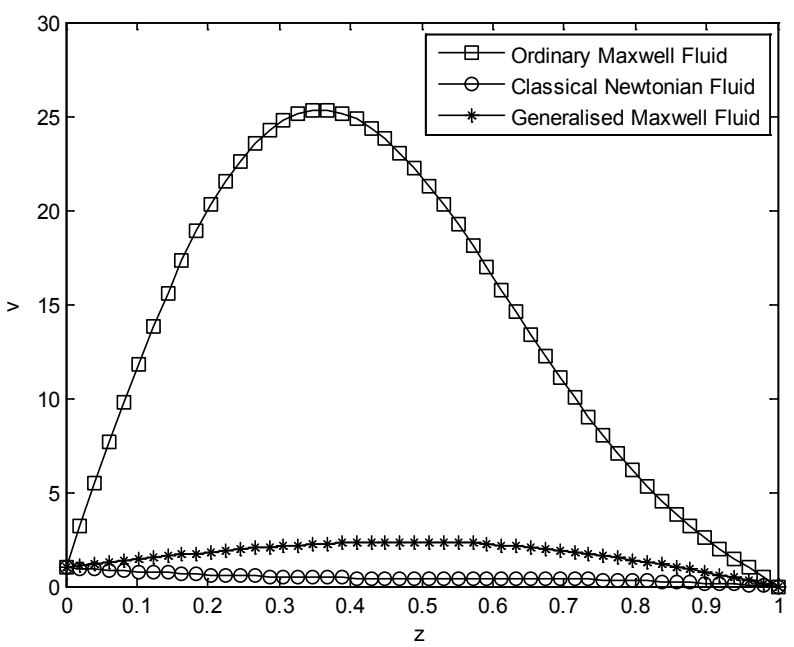

Figure 8. The velocity profile is depicted against the distance from the lower disk along the direction of the common axis of rotation for three cases. Case-I: Ordinary Maxwell Fluid, $\alpha=\beta=1.0, \zeta=0.03, \eta=0.02, s=0.2, t=0.2$; Case-II: Classical Newtonian Fluid. $\alpha=0.0, \beta=1.0, t=0.2$, $\zeta=0.03, \eta=0.02, s=0.2$; Case-III: Generalised Maxwell Fluid. $\alpha=0.2, \beta=0.8, t=0.2, \zeta=0.03, \eta=0.02, s=0.2$.

the lower disk) at which the velocity gradient is zero. The velocity curves for Classical Newtonian and Generalised Maxwell Fluids are almost parallel to the horizontal axis compared to the velocity curve for the Ordinary Maxwell Fluid. It can be seen that the velocity curve for the Generalised Maxwell Fluid has a point of local maximum.

\section{REFERENCES}

[1] T. Von Kármán, "Über Laminare und Turbulente Reibung," Zeitschrift für Angewandte Mathematik und Mechanik, Vol. 1, No. 4, 1921, pp. 233-255. doi:10.1002/zamm.19210010401

[2] W. G. Cochran, "The Flow Due to a Rotating Disc," Mathematical Proceedings of the Cambridge Philosophical Society, Vol. 30, No. 3, 1934, pp. 365-375. doi:10.1017/S0305004100012561

[3] M. A. Hossain and A. F. M. A Rahman, "The Steady Flow between Two Porous Rotating Discs in Presence of Transverse Magnetic Field," Indian Journal of Pure and Applied Mathmatics, Vol. 15, No. 2, 1984, pp. 187-194.

[4] M. A. Hossain, A. Hossain and M. Wilson, "Unsteady Flow of Viscous Incompressible Fluid with TemperatureDependent Viscosity Due to a Rotating Disc in Presence of Transverse Magnetic Field and Heat Transfer," International Journal of Thermal Sciences, Vol. 40, No. 1, 2001, pp. 11-20. doi:10.1016/S1290-0729(00)01183-2

[5] T. Wenchang, P. Wenxiao and X. Mingyu, "The Unsteady Flows of a Visco-Elastic Fluid with the Fractional Maxwell Model between Two Parallel Plates," International Journal of Non-Linear Mechanics, Vol. 38, No. 5, 2003, pp. 645-650. doi:10.1016/S0020-7462(01)00121-4

[6] S. L.Maji, N. Ghara, R. N. Jana and S. Das, "Unsteady 
MHD Flow between Two Eccentric Rotating Disks," Journal of Physical Science, Vol. 13, 2009, pp. 87-96.

[7] Y. Liu, L. Zheng, X. Zhang and F. Zong, "The Oscillating Flows and Heat Transfer of a Generalized Oldroyed-B Fluid in Magnetic Field," International Journal of Applied Mathematics, Vol. 40, 2010.

[8] M. Kempegowda and P. M. Balagondar, "The Exact So- lutions of Non-Newtonian Fluid Flow between Two Moving Parallel Disks and Stability Analysis," Applied Mathrmatical Sciences, Vol. 6, No. 37, 2012, pp. 1827-1835. 